\title{
Oral stimulation techniques in preterm infants - International research challenges ${ }^{\text {th }}$
}

\author{
Zelda Greene $^{\mathrm{a}, *}$, Colm P.F. O’Donnell ${ }^{\mathrm{b}}$, Margaret Walshe ${ }^{\mathrm{c}}$ \\ a Speech and Language Therapy Department, Our Lady's Children's Hospital, Crumlin, Dublin 12, Ireland \\ ${ }^{\mathrm{b}}$ Neonatology Department, The National Maternity Hospital, Holles Street, Dublin 2, Ireland \\ ${ }^{\mathrm{C}}$ Clinical Speech and Language Studies, Trinity College Dublin, Dublin 2, Ireland
}

Available online 23 April 2013

\author{
KEYWORDS \\ Preterm; \\ Infant feeding; \\ Oral stimulation; \\ Intervention; \\ Sucking; \\ Gavage feeds
}

\begin{abstract}
There has been a significant increase in the survival of preterm infants in recent years. These infants often face difficulty acquiring the complex set of skills required for exclusive oral feeding due to a multiplicity of factors. This paper discusses the theory underlying the use of oral stimulation interventions with the preterm infant, and their role in facilitating the transition from tube (gavage) feeds to exclusive oral feeding and ultimately discharge from the Neonatal Intensive Care Unit. Oral stimulation interventions are defined and the range of interventions described in the current literature is examined. The challenges that exist in deciphering the research evidence supporting their use is explored and directions for future research are provided.
\end{abstract}

(c) 2013 Neonatal Nurses Association. Published by Elsevier Ltd. All rights reserved.

\section{Introduction}

The complex skills, required for the development of sucking and feeding in infants, have been well documented (Bingham et al., 2010; Dodrill et al., 2008a; Pickler et al., 2006; Poore and Barlow,

\footnotetext{
Supported by the Health Research Board of Ireland Cochrane Fellowship Scheme.

* Corresponding author. Tel.: +35314096198.

E-mail addresses: zeldagreene@gmail.com, zelda.greene@ olchc.ie (Z. Greene).
}

2009). For the preterm infant acquisition of these skills means dealing with many added challenges depending on the extent of the prematurity and the myriad of accompanying co-morbidities and experiences encountered on the journey through the Neonatal Intensive Care Unit (NICU). Many multidisciplinary team members are involved in helping the preterm infant navigate through the NICU and often the ability to feed orally determines discharge from the unit. Early discharge from NICU has financial implications for healthcare providers. Figures from the United States estimate 
that a 3-day decrease in hospital stay for this population could save more than 2 billion dollars annually (Lessen, 2011). This fact alone makes it tempting for many financially stretched health care providers to look at the implementation of interventions in this population that will facilitate early oral feeding. Early exclusive oral feeding has thus become an important focus of intervention for the multidisciplinary team.

Oral stimulation interventions are popular and many are described in the literature, all developed to promote early oral feeding (See Table 1). Pinelli and Symington (2005) report that non nutritive sucking (NNS) was found to decrease significantly the length of hospital stay for preterm infants and had a positive influence on transition from tube to bottle feeds with better bottle feeding performance. Another systematic review has explored direct oral stimulation techniques (Arvedson et al., 2010). This concluded that although oral motor interventions show promise for enhancing feeding and swallowing in preterm infants, there remains no clear direction for clinical practice.

Our team has set out to address specific key questions regarding oral interventions with preterm infants and to provide some evidence for both clinical practice and research in the area. A protocol for this review is published (Greene et al., 2012) and a Cochrane systematic review is underway. In conducting this review we have encountered two key challenges:

1. the construction of a theoretical framework to support the practice of oral motor interventions.

2. negotiating the published literature due to variations in terminology used and lack of clear protocols for practice internationally.
Oral interventions for preterm infants: the theoretical framework for practice

\section{The development of oral feeding in the preterm infant}

Oral feeding is a complex skill requiring the integration of breathing, sucking and swallowing in the context of overall motor stability and incoming sensory stimuli (Arvedson et al., 2010; da Costa et al., 2010a). It depends upon brainstem central pattern generators whose activity is increasingly influenced by chemosensory and oral tactile input (Amaizu et al., 2008; Bingham, 2009). For the preterm infant the transition to oral feeding from gavage (tube) feeding can be a challenge as it requires ability to coordinate the muscles of the jaw, lips, tongue, palate and pharynx, upper trunk and respiratory systems in order to support a safe swallow. It is also dependent on normal sensory functioning, for example the presence of reflexes of rooting, gagging, swallowing, as well as intra-oral and pharyngeal sensation. Therefore, both sensory and motor systems must reach a critical stage of development for the infant to be able to feed orally.

The developmental stages of sucking in low birth weight preterm infants during bottle feeding have been described (Amaizu et al., 2008; Bingham et al., 2010; Dodrill et al., 2008a; Neiva and Leone, 2007). Varying components of sucking physiology such as sucking amplitude, rate, pressure intensity, timing of sucking cycles, sucking proficiency and efficiency appear to mature over time although at varying rates depending on several motor and sensory factors (Bingham et al., 2010; Poore et al., 2008; Matsubara et al., 2005). Preterm infants frequently remain in hospital for protracted periods as they learn to transition from gavage to oral feeds, mastering these skills.

Table 1 Oral stimulation interventions.

Named intervention
Perioral stimulation ranging from 5 to 15 min
Patterned orocutaneous therapy - 'pulsating pacifier'
Perioral stimulation followed immediately
by pacifier for NNS
Pacifiers during gavage feeds
Sweet pacifier
Semi-demand gavage feeds \& pacifier during feeds
Music therapy - pacifier activated lullaby
Oral support during feeding
Tactile/kinesthetic whole body stimulation

\section{Clinical trials}

Boiron et al. 2009/2007; Harding et al., 2006; Gaebler and Hanzlik, 1996; Neiva and Leone, 2006/2007; Lessen, 2011

Poore et al., 2008/Barlow et al., 2008

Fucile et al., 2012/2011/2005/2002;

Pimenta, 2008; Rocha et al., 2007

Yildiz and Arikan, 2011/Field et al., 1982;

Mattes et al., 1996

McCain et al., 2001/McCain and Gartside, 2002

Standley et al., 2010/Yildiz et al., 2011

(Boiron et al., 2009)

(Fucile et al., 2011/2012; Bragelien et al., 2007) 
However, the development of feeding in the preterm infant can be complicated by the presence of a multiplicity of other factors, which must be taken into consideration for holistic management.

\section{Factors influencing the development of oral feeding in the preterm infant}

In the preterm population, the existence of several comorbidities e.g. respiratory disease, can impose limits on the opportunities for sucking. This can deprive the infant of necessary oral sensori-motor experiences during a critical period of brain development when central patterning of suck and feeding skills are being refined (da Costa et al., 2010b; Mizuno et al., 2007; Stumm et al., 2008). Other medical interventions used in preterm infants (e.g. prolonged intubation, continuous positive airway pressure, nasal cannulation, regular oropharyngeal, nasal or tracheal suction) may compound negative responses by the infant to oral feeding trials (Bingham, 2009; Dougherty and Luther, 2008; Jadcherla et al., 2010). General factors such as prefeeding behavior, state and feeding experience also influence feeding performance in this population (Dodrill et al., 2008a; Pickler et al., 2006). Some intervention that would ameliorate the impact of negative feeding experiences, provide some positive oral motor sensory stimulation and expedite the transition from tube to oral feeding would therefore seem important.

\section{Factors influencing the selection of an oral intervention}

Readiness to feed in an important construct in the argument for oral motor interventions. Empirically derived guidelines for either starting or progressing oral feeds are lacking (Crowe et al., 2012; Pickler et al., 2006). The criteria for making judgments on readiness to feed can vary between centers and seems dependent on a range of factors such as age, weight, oral motor skills, feeding techniques, and feeding experience (Crowe et al., 2012; Dodrill et al., 2008b; Zimmerman and Barlow, 2009). Using age alone as a deciding factor for feeding is a crude measure as it does not consider other feeding readiness indices such as respiratory state, physiologic stability, self regulation skills etc. To help clinicians determine readiness for feeding and suitability for oral trials, a number of authors have provided some direction. For example, the Early Feeding Skills Assessment for preterm infants (Thoyre et al., 2005) and the more recent SOFFI - Supporting Oral Feeding in
Fragile Infants (Ross and Philbin, 2011a,b) provide some direction for evaluation. Other studies incorporate observation of infant behavior before feeding (Als et al., 2003). Cue based and semi demand feeding protocols have been described which rely on observations of the infant behavior, requiring clinicians to seek cues indicating feeding readiness instead of administering gavage feeds on a strict four hourly basis (McCain and Gartside, 2002). A Cochrane review in this area revealed limited evidence that this approach allows earlier attainment of oral feeds and earlier hospital discharge (McCormick et al., 2010).

Having determined the readiness to feed, the next challenge is to determine which specific intervention can facilitate sucking and feeding development to expedite the transition to oral feeding. The clinician is faced with a range of approaches (See Table 1). In broad terms these interventions are designed to decrease oral hypersensitivity, improve range of motion and strength of muscles for sucking (Fucile et al., 2002), increase oral motor organization (CaseSmith, 1989) and activate reflex behaviors that facilitate nutritive sucking (Leonard et al., 1980). In general the techniques aim to normalize sensation by restoring reflexes and in turn elicit normal oral movements of lips, tongue, jaw and pharynx for sucking and swallowing development. The decision on which approach is most effective lies in deciphering the evidence.

\section{Challenges in reviewing the evidence for oral motor interventions}

\section{Terminology: Who is the study about?}

Preterm infants are typically defined as those born before 37 weeks postmenstrual age (PMA) i.e. up to 36 weeks and 6 days. The definition of preterm can vary (see Table 2 ). When definitions are used, they can lead to further confusion as a variety of terms are used to describe populations in studies on preterm infants (Table 2). The American Academy of Pediatrics (2004) recognized this problem over a decade ago with a call for consistency in the use of definitions to describe the length of gestation and age in neonates. Many studies fail to differentiate between early (EPT) and late (LPT) preterm infants and again there is confusion on these definitions. Some studies define LPT as $34-36$ weeks gestational age with EPT preterm infants classified those $<34$ weeks gestational age while others (Gunville et al., 2010) 


\begin{tabular}{|c|c|}
\hline Term & Definition \\
\hline $\begin{array}{l}\text { Gestational } \\
\text { age/menstrual } \\
\text { age }\end{array}$ & $\begin{array}{l}\text { Time elapsed between the } \\
\text { first day of the last normal } \\
\text { menstrual period and the } \\
\text { day of delivery }\end{array}$ \\
\hline $\begin{array}{l}\text { Chronological } \\
\text { age/postnatal } \\
\text { age }\end{array}$ & Time since birth \\
\hline $\begin{array}{l}\text { Postmenstrual } \\
\text { age (PMA) }{ }^{\mathrm{a}}\end{array}$ & $\begin{array}{l}\text { Time between the first day } \\
\text { of the last menstrual period } \\
\text { and birth (gestational age) } \\
\text { plus the time elapsed after } \\
\text { birth (chronological age). }\end{array}$ \\
\hline 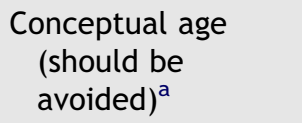 & $\begin{array}{l}\text { Time elapsed between the } \\
\text { day of conception and the } \\
\text { day of delivery }\end{array}$ \\
\hline $\begin{array}{l}\text { Corrected age } \\
\text { (use after }^{\text {perinatal period) }}{ }^{a}\end{array}$ & $\begin{array}{l}\text { Chronological age reduced by } \\
\text { the number of weeks born } \\
\text { before } 40 \text { weeks of gestation. }\end{array}$ \\
\hline
\end{tabular}

define EPT as $<32$ weeks' gestational age and LPT as $>32$ weeks' gestation age at birth.

The implications are that it is often impossible to make an informed judgment on the efficacy and effectiveness of interventions used for the populations described or make comparisons to similar populations.

\section{Interventions: what is the intervention?}

The intervention itself is not always clearly defined in the research literature. For the purposes of our Cochrane review we define oral stimulation as: 'the sensory stimulation to or manipulated actions of the lips, jaw, tongue, soft palate, pharynx, larynx, and respiratory muscles before or during either nutritive sucking (NS) or nonnutritive (NNS) events, intended to influence the oropharyngeal and respiratory sensorimotor mechanisms in order to improve function for sucking and feeding in preterm infants' (Greene et al., 2012 p. 2). We determined that stimulation of NNS could be considered an oral stimulation intervention and that specific oral stimulation interventions could include activities to develop both NNS which involves use of a pacifier before or during tube or bottle feeds and/or direct oral or peri-oral stimulation techniques to facilitate eventual NS.

However, studies are not always clear about what exactly the intervention involved, who provided the intervention, the intensity of the intervention, and the context in which it was delivered (Table 1). Several authors describe more than one intervention (e.g. perioral stimulation program followed by a period of nonnutritive sucking on pacifier) but fail to determine which aspects of this intervention 'package' could be contributing to the eventual outcomes.

There are many other factors that augment the intervention provided making it difficult to discern if it is the intervention itself or the context in which the intervention is delivered that is important. Environmental and physical modifications such as eliminating external stimuli (e.g. light, noise, other sensory experiences) during feedings, using therapeutic nipples on bottles to manipulate milk flow rate, positioning and swaddling of the infant to support the motor and sensory system and promote flexion are all believed to prepare the infant for the feeding situation (Ross and Philbin, 2011a,b). Simple facts like holding the infant during feeding versus infant positioned supine in the incubator are important as they create two separate feeding environments for the infant, which may modulate feeding behavior. These modifications ensure that infants are suitably calm, alert and ready to attempt oral feeding. Direct replication of the intervention can be difficult with variations in research and clinical practice evident from the studies.

Indeed there are a selection of studies testing a range of other interventions which appear to have a positive impact on feeding skill and efficiency by using methods such as cross cut nipple teats (Chang et al., 2007), a controlled flow vacuum bottle system (Fucile et al., 2009), spoon feeding (Kumar et al., 2010), Auditory, Tactile, Visual and Vestibular (ATVV) interventions (White-Traut et al., 2002), breast milk odor (Yildiz et al., 2011) or music therapy (Vianna et al., 2011). Do these interventions come under the umbrella of oral stimulation intervention as they share similar outcomes? Perhaps our overriding definition of 'Oral stimulation intervention' needs to be more carefully defined to reflect the continuum of practices reported.

\section{Outcome of interventions: What to measure?}

The outcome measures for the interventions in the published literature vary. Our review is concerned specifically with outcome measures that signify improvement in oral feeding ability and oromotor function of the preterm infant and that reduce NICU and/or overall hospital stay. Suggestions for outcome measures in this population are provided in Table 3. Potential adverse outcomes associated with oral stimulation interventions also need to be documented with careful monitoring of events 
Table 3 Suggested outcome measures for oral interventions.

\begin{tabular}{|c|c|}
\hline Primary outcomes & Measurement \\
\hline Time taken to achieve exclusive oral feeding & $\begin{array}{l}\text { Number of days from commencement of intervention } \\
\text { to first day of exclusive oral feeding }\end{array}$ \\
\hline $\begin{array}{l}\text { Time taken to transition from tube } \\
\text { to full oral feeds }\end{array}$ & $\begin{array}{l}\text { Number of days from commencement of intervention } \\
\text { to first day of exclusive oral feeding }\end{array}$ \\
\hline Total hospital stay & $\begin{array}{l}\text { Number of days of total hospital admission including } \\
\text { days in hospital post NICU discharge }\end{array}$ \\
\hline Duration of parenteral nutrition & Number of days on parenteral nutrition \\
\hline Maturation in sucking strength & $\begin{array}{l}\text { Measured by e.g. rate of milk intake ( } \mathrm{ml} / \mathrm{min}) \text {; } \\
\text { suction amplitude (mmHg/sucks per minute); } \\
\text { objective measures arising from digital } \\
\text { equipment/suck transducers assessing suck ability }\end{array}$ \\
\hline Secondary outcomes & Measurement \\
\hline Exclusive oral feeding at term corrected age, & $\begin{array}{l}\text { Taking eight feedings per day for } 2 \text { consecutive days } \\
\text { (Fucile et al., 2002) or able to take more than } 80 \% \\
\text { of the prescribed total fluid intake orally in a } \\
24 \mathrm{~h} \text { period (Premji et al., 2004) }\end{array}$ \\
\hline $\begin{array}{l}\text { Exclusive direct breastfeeding at } \\
\text { term corrected age, }\end{array}$ & $\begin{array}{l}\text { Full oral intake delivered by breast feeding and } \\
\text { measured qualitatively using a breast feeding } \\
\text { assessment } \\
\text { tool e.g. LATCH breastfeeding charting system }\end{array}$ \\
\hline Any direct breastfeeding at term corrected age, & $\begin{array}{l}\text { At least one feed delivered via breast in } 24 \mathrm{~h} \text { time } \\
\text { period }\end{array}$ \\
\hline Growth measures & $\begin{array}{l}\text { Weight gain }(\mathrm{g} / \mathrm{kg} / \text { day }) \text {, Length of body }(\mathrm{cm} / \text { day }) \text {, } \\
\text { Headcircumference }(\mathrm{cm} / \text { day })\end{array}$ \\
\hline Developmental outcomes & $\begin{array}{l}\text { Ascertained by a validated instrument at } \\
12-18 \text { months }\end{array}$ \\
\hline Family satisfaction with intervention, & Satisfaction questionnaire/survey \\
\hline Non-compliance with intervention. & Observation, caregiver report \\
\hline
\end{tabular}

such as sepsis, oral infection, oral trauma, apnea or bradycardia episodes that require intervention from the caregiver (e.g. stimulation, oronasal suction, increase in delivery of oxygen, assisted ventilation) or increase in salivary flow (as measured by the presence of saliva beyond the level of the lips), oxygen dependence at 36 weeks PMA, or death during initial hospital stay.

\section{Follow up periods after intervention}

More longitudinal studies are required to determine the efficacy of an intervention. Many studies fail to consider outcomes beyond hospital discharge. Gunville et al. (2010) found that children born preterm comprise a substantial proportion of admissions to the Pediatric Intensive Care Unit (PICU) for respiratory illness in the first years of life. As a result, they utilize considerably more hospital resources and incur more expense than full term infants. Since feeding and swallowing difficulties can be strongly associated with respiratory difficulties, longer-term outcomes for oral interventions should include respiratory outcomes as well as feeding behaviors, weaning difficulties, and feeding aversions (Samara et al., 2010).

\section{Discussion}

Undoubtedly the argument for oral interventions to improve feeding in preterm infants will remain debated for some time to come. We believe that without more robust well-designed research in the area, as multidisciplinary team members, we may well be misdirecting our efforts in with this population. More multi-center RCTs are required that conform to CONSORT guidelines of reporting (Schulz et al., 2010). Clear descriptions of populations selected with precise definitions of the characteristics of the population, accurate recording of the infant's readiness to feed, detailed description of the intervention itself, its method (frequency, intensity and duration) and context of delivery with valid and reliable outcome measures recorded are required. We suggest both primary and secondary outcomes with long term follow up of infants' feeding behaviors post follow discharge. We propose that a range 
of time frames be reported, for example, immediate change, medium term change (6-12 months) and/or long-term change (12 months + ).

Small intervention studies that are well designed and accurately reported will lend themselves more readily to meta-analysis. Only with this evidence, will we then be able to deliver comprehensive cost effective services to this population.

\section{Conflict of interest statement}

\section{Authors have no conflicts of interest to declare.}

\section{Acknowledgment}

Zelda Greene is supported by the Health Research Board of Ireland.

\section{References}

Als, H., Gilkerson, L., Duffy, F.H., Mcanulty, G.B., Buehler, D.M., Vandenberg, K., Sweet, N., Sell, E., Parad, R.B., Ringer, S.A., Butler, S.C., Blickman, J.G., Jones, K.J., 2003. A three centre randomised controlled trial of individualized developmental care for very low birth weight preterm infants: medical, neurodevelopmental, parenting and caregiving effects. Journal of Developmental and Behavioural Pediatrics 24 (6), 399-408.

American Academy of Pediatrics, 2004. Age terminology during the perinatal period. Pediatrics 114, 1362-1364.

Amaizu, N., Shulman, R.J., Schanler, R.J., Lau, C., 2008. Maturation of oral feeding skills in preterm infants. Acta Paediatrica 97 (1), 61-67.

Arvedson, J., Clark, H., Lazarus, C., Schooling, T., Frymark, T., 2010. Evidence-based systematic review: effects of oral motor interventions on feeding and swallowing in preterm infants. American Journal of Speech-Language Pathology 19 (4), 321-340.

Barlow, S.M., Finan, D.S., Lee, J., Chu, S., 2008. Synthetic orocutaneous stimulation entrains preterm infants with feeding difficulties to suck. Journal of Perinatology 28 (8), $541-548$.

Bingham, P.M., 2009. Deprivation and dysphagia in premature infants. Journal of Child Neurology 24 (6), 743-749.

Bingham, P.M., Ashikaga, T., Abbasi, S., 2010. Prospective study of non-nutritive sucking and feeding skills in premature infants. Archives of Disease in Childhood - Fetal and Neonatal Edition 95 (3), F194-F200.

Boiron, M., Da Nobrega, L., Roux, S., Saliba, E., 2009. Pharyngeal swallowing rhythm in response to oral sensorimotor programs in preterm infants. Journal of Neonatal Nursing 15 (4), 123-128.

Bragelien, R., Røkke, W., Markestad, T., 2007. Stimulation of sucking and swallowing to promote oral feeding in premature infants. Acta Paediatrica 96 (10), 1430-1432. (Oslo, Norway: 1992).

Case-Smith, J., 1989. Intervention strategies for promoting feeding skills in infants with sensory deficits. Occupational Therapy in Health Care 6 (2), 129-141.
Chang, Y., Lin, C., Lin, Y., Lin, C., 2007. Effects of single-hole and cross-cut nipple units on feeding efficiency and physiological parameters in premature infants. The Journal of Nursing Research: JNR 15 (3), 215-223.

da Costa, S.P., van der Schans, C.P., Boelema, S.R., van der Meij, E., Boerman, M.A., Bos, A.F., 2010a. Sucking patterns in fullterm infants between birth and 10 weeks of age. Infant Behavior and Development 33 (1), 61-67.

da Costa, S.P., van der Schans, C.P., Zweens, M.J., Boelema, S. R., van, d.M., Boerman, M.A., et al., 2010b. The development of sucking patterns in preterm, small-for-gestational age infants. The Journal of Pediatrics 157 (4), 603.

Crowe, L., Chang, A., Wallace, K., 2012. Instruments for assessing readiness to commence suck feeds in preterm infants: effects on time to establish full oral feeding and duration of hospitalisation. Cochrane Database of Systematic Reviews (4). http://dx.doi.org/10.1002/14651858. CD005586.pub2. Art. No.: CD005586.

Dodrill, P., Donovan, T., Cleghorn, G., McMahon, S., Davies, P.S. , 2008a. Attainment of early feeding milestones in preterm neonates. Journal of Perinatology 28 (8), 549-555.

Dodrill, P., McMahon, S., Donovan, T., Cleghorn, G., $2008 \mathrm{~b}$. Current management of transitional feeding issues in preterm neonates born in Queensland, Australia. Early Human Development 84 (10), 637-643.

Dougherty, D., Luther, M., 2008. Birth to breast - a feeding care map for the NICU: helping the extremely low birth weight infant navigate the course. Neonatal Network: NN 27 (6), 371-377.

Field, T., Ignatoff, E., Stringer, S., Brennan, J., Greenberg, R., Widmayer, S., et al., 1982. Nonnutritive sucking during tube feedings: effects on preterm neonates in an intensive care unit. Pediatrics 70 (3), 381-384.

Fucile, S., Gisel, E., Lau, C., 2002. Oral stimulation accelerates the transition from tube to oral feeding in preterm infants. The Journal of Pediatrics 141 (2), 230-236.

Fucile, S., Gisel, E.G., Lau, C., 2005. Effect of an oral stimulation program on sucking skill maturation of preterm in fants. Developmental Medicine and Child Neurology 47 (3), 158-162.

Fucile, S., Gisel, E., Schanler, R.J., Lau, C., 2009. A controlled-flow vacuum-free bottle system enhances preterm infants' nutritive sucking skills. Dysphagia. ISSN: 0179051X 24 (2), 145-151.

Fucile, S., Gisel, E.G., Mcfarland, D.H., Lau, C., 2011. Oral and non-oral sensorimotor interventions enhance oral feeding performance in preterm infants. Developmental Medicine and Child Neurology 53 (9), 829-835.

Fucile, S., McFArland, D.H., Gisel, K.G., Lau, C., 2012. Oral and non-oral sensorimotor interventions facilitate suck-swallowrespiration functions and their coordination in preterm infants. Early Human Development 8, 345-350.

Gaebler, C.P., Hanzlik, J.R., 1996. The effects of a prefeeding stimulation program on preterm infants. American Journal of Occupational Therapy 50 (3), 184-193.

Greene, Z., Walshe, M., O'Donnell, C.P.F., 2012. Effects of oral stimulation for oral feeding in preterm infants. Cochrane Database of Systematic Reviews (3). http://dx.doi.org/10. 1002/14651858.CD009720. Art. No.: CD009720.

Gunville, C.F., Sontag, M.K., Stratton, K.A., Ranade, D.J., Abman, S.H., Mourani, P.M., 2010. Scope and impact of early and late preterm infants admitted to the PICU with respiratory illness. The Journal of Pediatrics 157 (2), 209-214.

Harding, C.M., Law, J., Pring, T., 2006. The use of non-nutritive sucking to promote functional sucking skills in premature infants: an exploratory trial. Infant 2 (6), 238-243.

Jadcherla, S.R., Wang, M., Vijayapal, A.S., Leuthner, S.R., 2010. Impact of prematurity and co-morbidities on feeding 
milestones in neonates: a retrospective study. Journal of Perinatology 30 (3), 201-208.

Kumar, A., Dabas, P., Singh, B., 2010. Spoon feeding results in early hospital discharge of low birth weight babies. Journal of Perinatology: Official Journal of the California Perinatal Association 30 (3), 209-217.

Leonard, E.L., Trykowski, L.E., Kirkpatrick, B.V., 1980. Nutritive sucking in high-risk neonates after perioral stimulation. Physical Therapy 60 (3), 299-302.

Lessen, B.,S., 2011. Effect of the premature infant oral motor intervention on feeding progression and length of stay in preterm infants. Advances in Neonatal Care (Elsevier Science) 11 (2), 129-139.

Matsubara, M., Tamura, Y., Ruchala, P., 2005. Analysis of nutritive sucking function in very low and extremely low birthweight infants in Japan: a pilot study. Japan Journal of Nursing Science 2 (1), 3-7.

Mattes, R.D., Maone, T., Wager-Page, S., Beauchamp, G., Bernbaum, J., Stallings, V., et al., 1996. Effects of sweet taste stimulation on growth and sucking in preterm infants. Journal of Obstetric, Gynecologic, and Neonatal Nursing: JOGNN/NAACOG 25 (5), 407-414.

McCain, G.C., Gartside, P.S., 2002. Behavioral responses of preterm infants to a standard-care and semi-demand feeding protocol. Newborn \& Infant Nursing Reviews 2 (3), 187-193.

McCain, G.C., Gartside, P.S., Greenberg, J.M., Lott, J.W., 2001. A feeding protocol for healthy preterm infants that shortens time to oral feeding. Journal of Pediatrics 139 (3), 374-379.

McCormick, F.M., Tosh, K., McGuire, W., 2010. Ad libitum or demand/semi-demand feeding versus scheduled interval feeding for preterm infants. Cochrane Database of Systematic Reviews (2). http://dx.doi.org/10.1002/14651858. CD005255.pub3. Art. No.: CD005255.

Mizuno, K., Nishida, Y., Taki, M., Hibino, S., Murase, M., Sakurai, M., et al., 2007. Infants with bronchopulmonary dysplasia suckle with weak pressures to maintain breathing during feeding. Pediatrics 120 (4), e1035-e1042.

Neiva, F.C.B., Leone, C.R., 2006. Sucking in preterm newborns and the sucking stimulation. Pró-Fono: Revista De Atualização Científica 18 (2), 141-150.

Neiva, F.C.B., Leone, C.R., 2007. Development of sucking rhythm and the influence of stimulation in premature infants. PróFono: Revista De Atualização Científica 19 (3), 241-248.

Pickler, R.H., Best, A.M., Reyna, B.A., Gutcher, G., Wetzel, P.A. , 2006. Predictors of nutritive sucking in preterm infants. Journal of Perinatology: Official Journal of the California Perinatal Association 26 (11), 693-699.

Pimenta, H.P., Moreira, M.E.L., Rocha, A.D., Gomes Jr., S.C., Pinto, L.W., Lucena, S.L., 2008. Effects of non-nutritive sucking and oral stimulation on breastfeeding rates for preterm, low birth weight infants: a randomized clinical trial. Jornal de Pediatria 84 (5), 423-427.

Pinelli, J., Symington, A., 2005. Non-nutritive sucking for promoting physiologic stability and nutrition in preterm infants. Cochrane Database of Systematic Reviews (Online) (4), CD001071.

Poore, M.A., Barlow, S.M., 2009. Suck predicts neuromotor integrity and developmental outcomes. Perspectives on Speech Science and Orofacial Disorders 19 (1), 44-51.
Poore, M., Zimmerman, E., Barlow, S.M., Wang, J., Gu, F., 2008. Patterned orocutaneous therapy improves sucking and oral feeding in preterm infants. Acta Paediatrica 97 (7), 920-927.

Premji, S.S., McNeil, D.A., Scotland, J., 2004. Regional neonatal oral feeding protocols: changing the ethos of feeding preterm infants. Journal of Perinatal and Neonatal Nursing 18 (4), 371-384.

Rocha, A.D., Moreira, M.E.L., Pimenta, H.P., Ramos, J.R.M., Lucena, S.L., 2007. A randomized study of the efficacy of sensory-motor-oral stimulation and non-nutritive sucking in very low birthweight infant. Early Human Development 83 (6), 385-388.

Ross, E.S., Philbin, M.K., 2011a. The SOFFI reference guide: text, algorithms, and appendices A manualized method for quality bottle-feedings. Journal of Perinatal \& Neonatal Nursing 25 (4), 360-380.

Ross, E.S., Philbin, M.K., 2011b. Supporting oral feeding in fragile infants an evidence-based method for quality bottlefeedings of preterm, ill, and fragile infants. Journal of Perinatal \& Neonatal Nursing 25 (4), 349-357.

Samara, M., Johnson, S., Lamberts, K., Marlow, N., Wolke, D., 2010. Eating problems at age 6 years in a whole population sample of extremely preterm children. Developmental Medicine and Child Neurology 52, e16-e22.

Schulz, K.F., Altman, D.G., Moher, D., CONSORT Group, 2010. CONSORT 2010 statement: updated guidelines for reporting parallel group randomised trials. PLoS Med 7 (3).

Standley, J.M., Cassidy, J., Grant, R., Cevasco, A., Szuch, C., Nguyen, J., et al., 2010. The effect of music reinforcement for non-nutritive sucking on nipple feeding of premature infants. Pediatric Nursing 36 (3), 138-146.

Stumm, S.L., Barlow, S.M., Estep, M., Lee, J., Cannon, S., Carlson, J., et al., 2008. Respiratory distress syndrome degrades the fine structure of the non-nutritive suck in preterm infants. Journal of Neonatal Nursing 14 (1), 9-16.

Thoyre, S.M., Shaker, C.S., Pridham, K.F., 2005. The early feeding skills assessment for preterm infants. Neonatal Network 24 (3), 7-16.

Vianna, M.N.S., Barbosa, A.P., Carvalhaes, A.S., Cunha, A.J.L.A. , 2011. Music therapy may increase breastfeeding rates among mothers of premature newborns: a randomized controlled trial. Jornal de Pediatria 87 (3), 206-212.

White-Traut, R., Nelson, M.N., Silvestri, J.M., Vasan, U., Patel, M., Cardenas, L., 2002. Feeding readiness behaviors and feeding efficiency in response to ATVV intervention... auditory, tactile, visual and vestibular. Newborn \& Infant Nursing Reviews 2 (3), 166-173.

Yildiz, A., Arikan, D., 2011. The effects of giving pacifiers to premature infants and making them listen to lullabies on their transition period for total oral feeding and sucking success. Journal of Clinical Nursing 21 (5-6), 644-656.

Yildiz, A., Arikan, D., Gözüm, S., Taştekın, A., Budancamanak, I. , 2011. The effect of the odor of breast milk on the time needed for transition from gavage to total oral feeding in preterm infants. Journal of Nursing Scholarship: An Official Publication of Sigma Theta Tau International Honor Society of Nursing/Sigma Theta Tau 43 (3), 265-273.

Zimmerman, E.A., Barlow, S.M., 2009. The complexity of transitioning to oral feeds in preterm infants. Perspectives on Speech Science and Orofacial Disorders 19 (1), 52-57. 